\title{
artigo
}

\section{Tratamento de malária recorrente em gestante com cloroquina e repercussões na Região de Rorainópolis- RR}

\author{
Treatment of recurrent malaria in pregnant women with chloroquine and repercussions in the Region of Rorainópolis- RR \\ Tratamiento del paludismo recurrente en mujeres embarazadas con cloroquina y repercusiones en la Región de \\ Rorainópolis- RR
}

\section{RESUMO}

A malária continua sendo, entre as doenças causadas por protozoários. As mulheres grávidas são consideradas um grupo particularmente vulnerável à malária por alterar o seu estado de imunidade durante a gestação, tornando-as mais suscetíveis às alterações no curso da gravidez, aumentando o risco de formas complicadas da doença. 0 objetivo deste artigo é avaliar prescrições de antimaláricos segundo indicadores de qualidade e descrever relatos de eventos adversos entre gestantes com malária não complicada através do uso da medicação a base de cloroquina. Como metodologia foi adotado o estudo de caso exposto toda a conduta médica adotada e o tratamento clínico da paciente. Os resultados mostraram que a paciente demonstrou reações devido ao efeito colateral, porém o parto foi a termo sem intercorrências com o recém-nascido. Concluindo que lugares endêmicos da doença se faz necessário saber como tratar corretamente ajustando-se aos protocolos recomendados pelo Ministério da Saúde.
\end{abstract}

DESCRITORES: Malária; Complicações Infecciosas na Gravidez; Recidiva.

\begin{abstract}
Malaria remains among the diseases caused by protozoa. Pregnant women are considered a group particularly vulnerable to malaria because they change their immunity during pregnancy, making them more susceptible to changes in the course of pregnancy, increasing the risk of complicated forms of the disease. The objective of this article is to evaluate prescriptions for antimalarials according to quality indicators and to describe reports of adverse events among pregnant women with uncomplicated malaria through the use of chloroquine-based medication. As a methodology, the exposed case study was adopted, all the medical conduct adopted and the clinical treatment of the patient. The results showed that the patient showed reactions due to the side effect, but the delivery was at term without complications with the newborn. Concluding that endemic places of the disease it is necessary to know how to treat correctly, adjusting to the protocols recommended by the Ministry of Health.
\end{abstract}

DESCRIPTORS: Malaria; pregnancy Complications, Infectious; Recurrence.

\section{RESUMEN}

La malaria sigue siendo una de las enfermedades causadas por protozoos. Las mujeres embarazadas se consideran un grupo particularmente vulnerable a la malaria porque cambian su inmunidad durante el embarazo, haciéndolas más susceptibles a cambios en el curso del embarazo, aumentando el riesgo de formas complicadas de la enfermedad. El objetivo de este artículo es evaluar las prescripciones de antimaláricos de acuerdo con indicadores de calidad y describir informes de eventos adversos en mujeres embarazadas con malaria no complicada mediante el uso de medicamentos a base de cloroquina. Como metodología se adoptó el estudio de caso expuesto, toda la conducta médica adoptada y el tratamiento clínico del paciente. Los resultados mostraron que la paciente presentó reacciones por efecto secundario, pero el parto fue a término sin complicaciones con el recién nacido. Concluyendo que los lugares endémicos de la enfermedad es necesario saber tratar correctamente, ajustándose a los protocolos recomendados por el Ministerio de Salud.

DESCRIPTORES: Malaria; Complicaciones Infecciosas del Embarazo; Recurrencia.

RECEBIDO EM: 31/03/2021 APROVADO EM: 22/06/2021 


\section{Paula Layana Vieira Wanderley}

Especialista em Saúde da Família pela Universidade Federal do Rio Grande do Norte-UFRN (2020) Graduada em Medicina pela Faculdade de Ciências Biomédicas de Cacoal/RO-FACIMED (2018). Atualmente atua como Médica da Atenção Básica e na área da Clínica Médica.

ORCID: 0000-0002-4707-7529

\section{Carlos Eduardo Tortelli Veloso}

Especialista em Saúde da Família pela Universidade Federal do Rio Grande do Norte-UFRN (2020) Graduado em Medicina pela Faculdade de Ciências Biomédicas de Cacoal/RO-FACIMED (2018). Atualmente atua como Médico da Atenção Básica e na área da Clínica Médica.

ORCID: 0000-0003-1542-2858

\section{Tâmara Vieira Monção}

Graduanda em Medicina Universidade Federal de Roraima, UFRR ( $10^{\circ}$ semestre). ORCID: 0000-0002-7168-365X

\section{INTRODUÇÃO}

A malária é conhecida por ser uma doença popular na região amazônica devido a quantidade casos registrados anualmente. Trata-se de uma infecção parasitária ocasionada por um protozoário intracelular obrigatório do gênero Plasmodium, cuja transmissão é feita através da fêmea infectada do mosquito pertencente ao gênero Anopheles, ocorrendo quando este mosquito se alimenta de sangue num indivíduo ${ }^{1}$.

Os sintomas da malária são provocados quando ocorre a liberação de toxinas após o desenvolvimento do parasita nos estágios primários da doença. Os primeiros sintomas são inespecíficos e semelhantes aos de uma síndrome gripal, tais como: febre, calafrios e sudorese. Para confirmação da doença é realizado um exame de gota espessa através de lâmina microscópica. Nesta fase inicial, os pacientes podem ser facilmente tratados, com recuperação rápida e completa, porem se procurado tardiamente, o tratamento pode evoluir para a malária grave ${ }^{2}$.

Na gestação, é uma condição considerada potencialmente grave, que atinge a população do mundo inteiro, sendo estimado que a cada ano 50 milhões de mulheres grávidas são expostas ao risco desta infecção anualmente. As gestantes compõem um grupo particularmente vulnerável a malária devido a alteração no sistema imunológico 3 .

Dessa forma consiste no aumento dos riscos e complicações durante a gestação derivadas das alterações causadas pela malária, tais como anemia materna e óbito materno. prematuridade, baixo peso ao nascer, perda fetal e morte materna. E para o feto, a malária materna representa a ameaça de aborto e de parto prematuro, baixo peso ao nascer e crescimento intrauterino restrito ${ }^{4}$.

As principais estratégias no tratamento da malária em gestantes adotadas pelo Ministério da Saúde destacam-se o tratamento medicamentoso, orientado por meio do protocolo oficial sendo revisado periodicamente. $\mathrm{O}$ protocolo trata a organização de esquemas terapêuticos para todos os pacientes, dentre eles o da malária gestacional, variando de acordo com a espécie parasitária, a gravidade da doença e a presença de gestação, e outros fatores 5 .

No protocolo para as gestantes com malária não complicada pelo P. vivax, por exemplo, é recomendado o uso do medicamento a base de cloroquina como primeira escolha. $\mathrm{O}$ tratamento consiste na administração de 4 comprimidos no primeiro dia do início do protocolo e de 3 comprimidos nos demais dias, totalizando 3 dias de tratamento ${ }^{6}$.

No caso de infecções pelo P. falciparum com essa mesma condição, o tratamento consiste no uso de três comprimidos de sulfato de quinina ao dia por um período de sete dias. Outro tratamento possui o protocolo da administração de sulfato de quinina $30 \mathrm{mg} / \mathrm{kg} /$ dia durante 3 dias, associado a $20 \mathrm{mg} / \mathrm{kg}$ de clindamicina, quatro vezes ao dia, durante um período de cinco dias ${ }^{6}$.
O objetivo deste artigo é avaliar prescrições de antimaláricos segundo indicadores de qualidade descrevendo os relatos de eventos adversos entre gestantes com malária não complicada através do uso da medicação a base de cloroquina.

\section{MÉTODOS}

$\mathrm{O}$ presente artigo adotou o método qualitativo para o referencial teórico utilizado através de fontes bibliográficas a partir de artigos científicos, livros e protocolos fornecidos pelo Ministério da Saúde (MS), revistas cientificas, tais como Revista Saúde Coletiva (Baueri), Revista Brasileira de Ginecologia e Obstetrícia, Revista Brasileira de Epidemiologia, Revista da Sociedade Brasileira de Medicina Tropical dentre outros. Trata-se de um estudo de caso, com a descrição do diagnóstico e evolução da malária na gestação. A pesquisa foi realizada no ano de 2019 com duração de 07 meses.

Expondo os mecanismos da doença e dos tratamentos, a partir de estudos clínicos e laboratoriais utilizados anteriormente em outros casos, visto que traz grande relevância e contribuição cientifica, traz o tratamento de uma paciente gestante com diagnostico de malária. É feito o relato desde a primeira consulta, como protocolo utilizado e acompanhamento do processo de cura.

A coleta dos dados foi realizada na Unidade Básica de Saúde Gentil Carneiro, localizada no município de Rorainópolis 
- Roraima, através do prontuário médico da paciente, que autorizou a publicação dos seus dados clínicos para uso exclusivo de pesquisa cientifica através da assinatura do Termo Livre de Consentimento (TLC) resolução no ${ }^{\circ}$ 466/12 do Conselho Nacional de Saúde - CNS.

O limite temporal estabelecido foi a duração do acompanhamento das consultas de pré-natal e puerpério realizadas pela paciente durante o ano de 2019. A paciente apresentou sintomas semelhantes a malária sendo confirmado posteriormente através de exame. A doença se fez presente durante toda a gestação através de recorrentes episódios. Como caráter de inclusão para a pesquisa foi utilizado a quantidade de exames testados positivo para a malária durante a gestação, caracterizando como uma patologia recorrente que durou todo período gestacional.

Sabe-se que no período gestacional quando ocorre a gestante se infecta com a malária, o protocolo base de tratamento recomenda-se o uso do fármaco Cloroquina como primeira escolha. Nos resultados é apresentado o relato de caso juntamente com a conduta adotada durante a gestação e o período puerperal da paciente.

\section{RESULTADOS}

Paciente S.S.S, 32 anos, iniciou o Pré Natal de baixo risco no dia 06 de dezembro de 2018 com idade gestacional equivalente a 9 semanas, referindo-se ao dia da última menstruação (DUM) em 03 de outubro de 2018, residindo em área endêmica para doenças emergentes.

No dia 07 de janeiro de 2019, idade gestacional equivalente a 13 semanas e 5 dias, paciente veio em consulta relatando sintomas como, náuseas, febre e vômitos. Sendo encaminhada a realizar o exame de malária através da lâmina, o resultado foi positivo para vivax, A conduta adotada foi o uso da medicação cloroquina $150 \mathrm{mg}$ por 3 dias seguidos. $\mathrm{O}$ exame foi repetido no dia 28 de janeiro de 2019 e deu negativo.

No dia 06 de fevereiro de 2019 referiu em consulta médica apenas cefaleia, foi solicitado novamente gota espessa no dia
08 de fevereiro de 2019 positivo malária vivax novamente, estava com idade gestacional equivalente a 18 semanas e 2 dias foi tratada por obstetra da região com cloroquina semanal até dia 08 de março de 2019, parou devido a médica se mudar do município, durante esse período feito lâmina de malária no dia 19 de março de

Sabe-se que no período gestacional quando ocorre a gestante se infecta com a malária, o protocolo base de tratamento recomenda-se o uso do fármaco Cloroquina como primeira escolha. Nos resultados é apresentado o relato de caso juntamente com a conduta

\section{adotada durante}

a gestação e o período puerperal da paciente.
2019 - malária negativo, conduta foi fazer lâmina de malária todo mês.

Novamente durante a consulta do pré natal ocorrida em 15 de abril 2019 com I idade gestacional equivalente a 27 semanas e 5 dias, apresentou com lâmina resultado positivo para vivax, foi tratado com cloroquina por 3 dias, após isso foi iniciado novamente o tratamento de malária recorrente na atenção primária, no dia 10 de maio 2019 cloroquina semanal 150mg 02 comprimidos 1 vez por semana por 12 semanas, e solicitado lâmina de malária de 15/15 dias, e acompanhamento com consultas do pré natal de $15 / 15$ dias.

No dia 03 de junho 2019 com idade gestacional equivalente a 34 semanas e 5 dias, teve contraçôes e fortes dores, precisando ir para a emergência do município, onde foi diagnosticada com ameaça de parto prematuro, encaminhado para a maternidade localizada no município de Boa Vista, referência da região, foi feito corticoide e nifedipino, e obteve a estabilização do quadro, ficou 5 dias internada, durante a internação tomou dose semanal de cloroquina e obteve alta mantendo a prescrição da cloroquina.

Compareceu para a consulta dia 11 de junho 2019, idade gestacional equivalente a 35 semanas e 6 dias compareceu em consulta, referindo o quadro de ameaça de parto prematuro e fraqueza intensa, a conduta foi encaminhar ao alto risco devido complicações de malária na gestação, porém paciente devido a condições sociais não pode ir para a referência em boa vista. Paciente continuou com acompanhamento semanal.

No dia 18 de junho de 2019 com idade gestacional equivalente a 36 semanas e 6 dias, queixando-se de mal estado geral, náuseas e diarreia há 2 dias, repetida lâmina de malária dando negativo, no momento estava na sétima semana do tratamento.

E por fim, no dia 04 de julho de 2019 aconteceu o parto cesariano, com I idade gestacional equivalente a 39 semanas e 1 dia. A cesárea ocorreu sem intercorrências, o recém-nascido não apresentou nenhum sintoma, nasceu bem, feito lâmina de malária em gestante e no $\mathrm{RN}$, os dois dando 
negativo, exames do $\mathrm{RN}$ sem alterações, em visita domiciliar puérpera e $\mathrm{RN}$ encontravam-se sem alterações ao exame físico, e sem queixas.

\section{DISCUSSÃO}

O protocolo utilizado na gestação está amparado pelo protocolo de estratégias adotadas pelo Programa Nacional de Controle da Malária ${ }^{5}$. O tratamento oportuno e adequado dos casos é uma das principais estratégias adotadas pelo Programa Nacional de Controle da Malária. Neste estudo verificou-se o uso do fármaco cloroquina, em monoterapia, uma das variedades de esquemas antimaláricos utilizados para o tratamento das gestantes, este medicamento é classificado na gestação como categoria $\mathrm{C}$, onde estudos em animais mostraram anormalidades nos descendentes, porém não há estudos em humanos, o risco para o bebê não pode ser descartado, mas uso da medicação ainda é o mais usado visto que o benefício potencial pode ser maior que os riscos ${ }^{7}$.

$\mathrm{O}$ uso da cloroquina foi predominante em toda a gestação devido os episódios recorrentes de queixas e exames positivos para a doença. Durante o tratamento a paciente apresentou reações devido ao efeito colateral do uso da cloroquina na gestação e em geral na gestante, como dores de cabeça, tonturas, cansaço, náuseas, vômitos, perda de apetite, desconforto abdominal. Os efeitos colaterais acometidos em pacientes gravidas são comuns e são descritos em toda a literatura ${ }^{8}$.

Uma das principais preocupações da malária na gestação é devido ser uma das causadoras de muito parto prematuro ou ameaça. No estudo de caso pode-se perceber através dos sintomas relatados que a paciente apresentou sintomas consiste com a ameaça do parto prematuro, sendo encaminhada para a Maternidade para tratamento e dessa forma o parto ocorreu dentro do período previsto sendo considerada uma gestação foi a termo ${ }^{4}$.

Apesar da literatura relatar casos clínicos ontem o recém-nascido pode desenvolver malária placentária ou ter sequelas acometidas durante o período da gestação em que houve a intercorrência da doença, o RN da paciente do estudo não foi atingido, sendo comprovado posteriormente através de exame e mantendo acompanhamento durante as consultas rotineiras do puerpério.

Esse relato de caso vai ser um acréscimo na literatura da eficiência do uso exclusivo de cloroquina no tratamento recorrente da malária na gestação. Devido à falta de especificidade de medicamentos de uso exclusivo na gestação pouco se fala sobre isso na literatura, sendo que, lugares endêmicos faz-se necessário saber como tratar corretamente esta doença afim de não causar nenhum prejuízo tanto para a mãe quanto ao bebê, evitando também que a gestante possa apresentar vários episódios durante este periodo. ${ }^{9}$

\section{CONCLUSÃO}

Como foi visto a malária é uma doença curável, após a identificação da espécie parasitária pelo exame laboratorial da gota espessa, deve ser imediatamente instituído o tratamento de acordo com os protocolos apresentados pelo Ministério da Saúde, dessa forma podendo evitar complicações ou intercorrências e o desenvolvimento da malária grave.

Vale ressaltar que é de maior importância que todos os profissionais de Saúde envolvidos no tratamento da malária como também no pré natal, orientem as pacientes gestantes quanto o tipo de medicamento que está sendo oferecido, à forma de ingeri-lo e os respectivos horários como também a importância de não interromper o tratamento medicamentoso.

Com isso este trabalho contribui para a conduta médica a ser utilizada no combate à malária na gestação elencando os principais cuidados no pré-natal. Espera-se, ainda, que a metodologia de avaliação utilizada possa ser empregada para o acompanhamento do tratamento na gestação para as demais endemias, sendo referência para o enfrentamento de malária sendo baseado no diagnóstico precoce e no tratamento.

\section{REFERÊNCIAS}

1. Reis, T. et al. Malaria: estudo retrospectivo de casos clínicos suspeito de infecção por Plasmodium sp (2010-2014). Boletim epidemiológico, v. 15, n. 2, 2016.

2. Ribeiro, E. et al.; Malária grave secundaria a co-infecção por P. falciparum e P. ovale. Galícia Clin, v. 74, n. 1, p.25-28, 2013.

3. Almeida LB, Barbosa MGV, Martinez-Espinosa FE. Malária em mulheres de idade de 10 a 49 anos, segundo o SIVEP - Malária, Manaus, Amazonas, 2003-2006. Rev Soc Bras Med Trop 2010; 43: 304-08.

4. CHAGAS, E. C. S. Malária durante a gravidez: efeito sobre o curso da gestação na região Amazônica. Dissertação de Mestrado. Manaus: Universidade do Estado do Amazonas, 2009.

5. Brasil. Ministério da Saúde. Guia prático de tratamento da malária no Brasil. Brasília: Ministério da Saúde; 2009.
6. Luiz, T. C. B; et al. Prescrições para o tratamento de malária não complicada em gestantes na Amazônia legal: evidências do projeto Mafalda. Rev. Bras. Epidemiol, v. 16, n.2, p.409-19, 2013.

7. Jarude R, Trindade R, JT-N. Malária em Grávidas de uma Maternidade Pública de Rio Branco (Acre, Brasil). Rev Bras Ginecol Obstet 2003; 25: 149-54.

8. Reiners AAO, Azevedo RCS, Ricci HA, Souza TG. Adesão e reações de usuários ao tratamento da malária: implicações para a educação em saúde. Texto \& Contexto Enfermagem. 2010; 19: 536-44.

9. Santos, R.C.S. Malária em gestantes atendidas no Hospital da Mulher Mãe Luzia (HMML), em Macapá, Amapá, no período de 2009 a 2010. 71f. Dissertação (Mestrado) Universidade Federal do Amapá, Macapá, 2011. 